\title{
EIGENVALUES OF ELLIPTIC BOUNDARY VALUE PROBLEMS WITH AN INDEFINITE WEIGHT FUNCTION
}

\author{
JACQUELINE FLECKINGER $^{1}$ AND MICHEL L. LAPIDUS ${ }^{2}$
}

ABStraCt. We consider general selfadjoint elliptic eigenvalue problems

$$
A u=\lambda r(x) u,
$$

\begin{abstract}
in an open set $\Omega \subset \mathbf{R}^{k}$. Here, the operator $A$ is positive and of order $2 m$ and the "weight" $r$ is a function which changes sign in $\Omega$ and is allowed to be discontinuous. A scalar $\lambda$ is said to be an eigenvalue of (P) if $A u=\lambda r u$-in the variational sense-for some nonzero $u$ satisfying the appropriate growth and boundary conditions. We determine the asymptotic behavior of the eigenvalues of $(\mathrm{P})$, under suitable assumptions. In the case when $\Omega$ is bounded, we assumed Dirichlet or Neumann boundary conditions. When $\Omega$ is unbounded, we work with operators of "Schrödinger type"; if we set $r_{ \pm}=\max ( \pm r, 0)$, two cases appear naturally: First, if $\Omega$ is of "weighted finite measure" (i.e., $\int_{\Omega}\left(r_{+}\right)^{k / 2 m}<+\infty$ or $\left.\int_{\Omega}\left(r_{-}\right)^{k / 2 m}<+\infty\right)$, we obtain an extension of the well-known Weyl asymptotic formula; secondly, if $\Omega$ is of "weighted infinite measure" (i.e., $\int_{\Omega}\left(r_{+}\right)^{k / 2 m}=+\infty$ or $\int_{\Omega}\left(r_{-}\right)^{k / 2 m}=+\infty$ ), our results extend the de Wet-Mandl formula (which is classical for Schrödinger operators with weight $r \equiv 1$ ). When $\Omega$ is bounded, we also give lower bounds for the eigenvalues of the Dirichlet problem for the Laplacian.
\end{abstract}

1. Introduction. The purpose of this paper is to provide estimates on the eigenvalues of "right nondefinite" elliptic boundary value problems $A u=\lambda r u$ in $\Omega \subset \mathbf{R}^{k}$ (with Dirichlet or Neumann boundary conditions) when the weight function $r$ is "indefinite" (i.e., changes sign in $\Omega$ ).

The main results concern the asymptotics of the eigenvalues for selfadjoint elliptic operators of order $2 m$. For an open set $\Omega$ which is not necessarily bounded, we extend the classical Weyl formula as well as its analogue, also called the de WetMandl formula for operators of Schrödinger type. We also obtain lower bounds for the eigenvalues of the Dirichlet problem for the Laplacian. These results extend the earlier ones obtained independently by Lapidus [La 1, La 2] and Fleckinger-E] Fetnassi [FlF 1, FlF 2].

Many nonlinear problems lead, after linearization, to elliptic eigenvalue problems with an indefinite weight function. (See, e.g., the survey paper by de Figueiredo

Received by the editors June 26, 1985.

1980 Mathematics Subject Classification. Primary 35P20, 35J20, 35J25, 35J35, 35J40, 35P15; Secondary 34B25, 35J10, 35P30, 47A70, 49G05.

Key words and phrases. Elliptic boundary value problems, indefinite weight function, asymptotic behavior of eigenvalues, lower bounds of eigenvalues, spectral theory, variational methods, operators of Schrödinger type.

${ }^{1}$ Research partially supported by the Centre National de la Recherche Scientifique and Argonne National Laboratory.

${ }^{2}$ Supported in part by a Grant from the Faculty Research and Innovation Fund at the University of Southern California and by Argonne National Laboratory. The second author would like to dedicate his research in this work to Odile. 
[dF] and the work of Hess and Kato $[\mathbf{H K}, \mathbf{H}]$.$) A vast literature in engineering,$ physics and applied mathematics deals with such problems arising, for instance, in the study of transport theory, reaction-diffusion equations, fluid dynamics, etc. (See, e.g., [DGHa, GN, LuR, LuW and KKLeZ].)

Most of the literature on this subject-studied since the end of the last century (Richardson $[\mathbf{R i}]$, Bôcher $[\mathbf{B o}]$, Hilbert $[\mathbf{H i}]$, etc.) -is concerned with the one dimensional case, which is still the object of active research. (See, for instance, $[\mathbf{K Z}$, Be].) To our knowledge, the first result in the multidimensional case is due to Holmgren (1904) [Ho]; he considers the Dirichlet problem $\Delta u+\lambda r(x, y) u=0$, on a bounded open set set $\Omega \subset \mathbf{R}^{2}$, when $r$ is continuous and changes sign; he proves in this case the existence of an infinite number of positive (and negative) eigenvalues which can be characterized by the "min-max principle". The asymptotic distribution of these eigenvalues has been established by Pleijel (1942) [Pl] for the Dirichlet and Neumann problems.

The spectral theory of similar abstract problems has been studied more recently by Weinberger $[\mathbf{W n}]$, and its application to the Dirichlet problem for an elliptic operator of order 2 by Manes and Micheletti [MM].

Pleijel's results on the asymptotic distribution of eigenvalues have been extended by many Soviet mathematicians during the 1970s. (See, for instance, the survey paper by Birman and Solomjak [BS 3], their book [BS 4] and the relevant references therein.) In most of these papers, the weight function need not be continuous. Birman and Solomjak [BS 1, BS 2], for example, obtain asymptotic estimates for bounded open sets and (possibly degenerate) selfadjoint elliptic operators of order $2 m$. Rozenbljum [Ro $\mathbf{1}$ ] extends these resuls to some problems defined on unbounded open sets such that $\int_{\Omega}|r|^{k / 2 m}<+\infty$.

Recently, unaware of Pleijel's work, Lapidus [La 1 ] determined the asymptotic behavior of the eigenvalues of the Laplacian with Dirichlet or Neumann boundary conditions on a bounded open set of $\mathbf{R}^{k}$ in the case when the weight function is not necessarily continuous; he also gives lower bounds having the correct "coupling constant behavior" for the eigenvalues of the Dirichlet problem. In some cases, he obtains estimates on the remainder term [La 2].

At about the same time, Fleckinger and El Fetnassi [FlF 1, FlF 2] proved an analogous asymptotic estimate for the Dirichlet problem corresponding to a general selfadjoint elliptic operator of order $2 m$ with a continuous weight function; asymptotics were also obtained for operators of "Schrödinger-type" on unbounded domains for a sufficiently smooth weight function.

The present paper extends these results: for general elliptic problems, we do not assume that the open set $\Omega$ is bounded or that the weight function $r$ is continuous; the latter is of interest in view of possible applications to nonlinear problems.

We now indicate how this work is organized. After having stated our main results in the next section, we present in $\S 3$ the prototypal example of the Laplacian on an open set of finite volume and give lower bounds for the eigenvalues of the corresponding Dirichlet problem. We then extend this study in $\S 4$ to general elliptic boundary value problems of order $2 m$ on an open set $\Omega$ (which is bounded for the Neumann problem). Finally, in $\S 5$, we determine the asymptotic distribution of the eigenvalues of certain Schrödinger operators on $\Omega \subset \mathbf{R}^{k}$ with Dirichlet boundary 
conditions; in this case, $\Omega$ is unbounded and we deal with the four different cases: $\int_{\Omega}\left(r_{ \pm}\right)^{k / 2 m}=+\infty$ or $\int_{\Omega}\left(r_{ \pm}\right)^{k / 2 m}<+\infty$.

Since the first version of this paper was submitted, the work of Birman-Solomjak and Rozenbljum on boundary value problems with indefinite weights was brought to our attention. In particular, there is some overlap between Theorem 4.1 herein and Theorem 2 in [BS 1 or BS 2], as well as Theorem 5.2 herein and Theorem 2 in [Ro 1]. However, our results go much further; Theorem 5.1 and Proposition 5.4, stated for unbounded open sets, appear to be entirely new as well as the possible combinations resulting from Theorems 5.1 and 5.2.

2. Notation and main results. We introduce some notation which will be used throughout the aricle:

Let $m$ and $k$ be integers $\geq 1$.

Let $\Omega$ be an open set in $\mathbf{R}^{k}$ with boundary $\partial \Omega$.

The interior (resp., the closure) of $Q \subset \mathbf{R}^{k}$ is denoted by $Q^{\circ}$ (resp., $\bar{Q}$ ); if, in addition, $Q$ is Lebesgue measurable, $|Q|$ stands for its Lebesgue $k$-dimensional measure or "volume" in $\mathbf{R}^{k}$.

If $R \subset Q$, the notation $Q \backslash R$ indicates the complement of $R$ in $Q$. $\Omega$.

For $j \in \mathbf{N}, C^{j}(\Omega)$ is the space of $j$ times continuously differentiable functions in

For $\alpha=\left(\alpha_{1}, \ldots, \alpha_{k}\right) \in \mathbf{N}^{k}, D^{\alpha}$ is the derivative of order $|\alpha|=\alpha_{1}+\cdots+\alpha_{k}: D^{\alpha}=$ $\partial^{|\alpha|} / \partial x_{1}^{\alpha_{1}} \cdots \partial x_{k}^{\alpha_{k}} ;$ if, in addition, $\xi=\left(\xi_{1}, \ldots, \xi_{k}\right) \in \mathbf{R}^{k}$, then $\xi^{\alpha}=\xi_{1}^{\alpha_{1}} \cdots \xi_{k}^{\alpha_{k}}$.

$H^{m}(\Omega)$ denotes the Sobolev space of functions $u \in L^{2}(\Omega)$ such that the distributional derivatives $D^{\alpha} u$ are in $L^{2}(\Omega)$ for $|\alpha| \leq m$ (see, e.g., [Ad]). Recall that $H^{m}(\Omega)$ is a Hilbert space for the norm

$$
\|u\|_{H^{m}(\Omega)}=\left(\int_{\Omega} \sum_{|\alpha| \leq m}\left|D^{\alpha} u(x)\right|^{2} d x\right)^{1 / 2} .
$$

$H_{0}^{m}(\Omega)$ is the subspace of $H^{m}(\Omega)$ obtained by completing $C_{0}^{\infty}(\Omega)$ with respect to the norm of $H^{m}(\Omega)$; here, $C_{0}^{\infty}(\Omega)$ denotes the space of continuously differentiable functions with compact support contained in $\Omega$.

Finally, if $f$ is a real-valued function defined on $\Omega$, we set $f_{+}=\max (f, 0)$ and $f_{-}=\max (-f, 0)$.

We can now state our hypotheses and present our main results:

(2.1) Let $A$ be a formally selfadjoint uniformly elliptic operator of order $2 m$ defined on $\Omega$ :

$$
A=\sum_{\substack{|\alpha| \leq m \\|\beta| \leq m}} D^{\alpha}\left(a_{\alpha \beta}(x) D^{\beta}\right)
$$

where $a_{\alpha \beta}=\overline{a_{\beta \alpha}}$ for $|\alpha| \leq m$ and $|\beta| \leq m$; we assume that $a_{0} \geq 0$ and-when $\Omega$ is bounded (resp., unbounded) $-a_{0} \in L^{k / 2 m}(\Omega)$ if $k \geq 2 m$ and $a_{0} \in L^{1}(\Omega)$ if $k<2 m$ [resp., $a_{0} \in L^{\infty}(\Omega)$ ]; moreover, $a_{\alpha \beta} \in L^{\infty}(\Omega)$ if $0<|\alpha|+|\beta| \leq 2 m$ and $a_{\alpha \beta} \in C^{0}(\bar{\Omega})$ if $|\alpha|+|\beta|=2 m$.

(2.2) Let $r$ be a measurable real-valued function on $\Omega$; we suppose that $\Omega_{+}=$ $\{x \in \Omega: r(x)>0\}$ and $\Omega_{-}=\{x \in \Omega: r(x)<0\}$ are of positive Lebesgue measure. In $\S \S 3-4$ (resp., §5), we also assume that $r \in L^{p}(\Omega)\left[\right.$ resp., $\left.r \in L_{\text {loc }}^{p}(\Omega)\right]$ with $p>k / 2 m$ if $k \geq 2 m$ and $p=1$ if $k<2 m$. Further, in $\S \S 3-4, \Omega$ is such that the imbedding of 
$H_{0}^{m}(\Omega)$ into $L^{2}(\Omega)$ is compact; this is always the case if $\Omega$ is bounded, $k<2 m$ or $\partial \Omega$ is smooth enough (see [Ad, Theorem 6.13, p. 151]).

We study the spectrum (i.e., the set of eigenvalues $\lambda$ ) of the homogeneous Dirichlet or Neumann boundary value problem (in the variational sense)

$$
A u=\lambda r u, \quad \text { in } \Omega .
$$

When $\Omega$ has finite Lebesgue measure, this spectrum is discrete and consists of a double sequence of real eigenvalues of finite multiplicity (one nonnegative and one negative)

$$
\cdots \leq \lambda_{n+1}^{-} \leq \lambda_{n}^{-} \leq \cdots \leq \lambda_{2}^{-} \leq \lambda_{1}^{-}<(0) \leq \lambda_{1}^{+} \leq \lambda_{2}^{+} \leq \cdots \leq \lambda_{n}^{+} \leq \lambda_{n+1}^{+} \leq \cdots,
$$

with $\left|\lambda_{n}^{ \pm}\right|$tending to $+\infty$ as $n$ tends to $\infty$ (each eigenvalue is repeated according to multiplicity). The same result holds, under appropriate assumptions, for an operator of "Schrödinger type" on $\Omega$ with Dirichlet boundary conditions.

Let $N^{+}(\lambda)$ [resp., $N^{-}(\lambda)$ ] be the number of nonnegative [resp., negative] eigenvalues $\lambda_{n}^{+}$less than $\lambda>0$ [resp., $\lambda_{n}^{-}$greater than $\lambda<0$ ].

Assume that $\Omega$ has weighted finite volume. (Of course, this assumption is satisfied if $\Omega$ is bounded.) If $\left|\Omega_{+}\right| \Omega_{+}^{\circ} \mid=0$ (this is the case, for example, if $r$ is continuous), we show for the Dirichlet problem that (see Theorem 4.1)

$$
N^{+}(\lambda) \sim \int_{\Omega_{+}}(\lambda r(x))^{k / 2 m} \mu_{\mathcal{A}}^{\prime}(x) d x, \quad \text { as } \lambda \rightarrow+\infty,
$$

where $\mu_{\mathcal{A}}^{\prime}(x)$ is the usual Browder-Gårding density on $\Omega$

$$
\mu_{\mathcal{A}}^{\prime}(x)=(2 \pi)^{-k} \int_{\left\{\xi \in \mathbf{R}^{k}: \mathcal{A}^{\prime}(x, \xi)<1\right\}} d \xi, \quad x \in \Omega,
$$

and $\mathscr{A}^{\prime}(x, \xi)$ is the symbol of the principal part of $\mathcal{A}$ :

$$
A^{\prime}(x, \xi)=\sum_{|\alpha|=|\beta|=m} a_{\alpha \beta}(x) \xi^{\alpha+\beta}, \quad \text { for }(x, \xi) \in \Omega \times \mathbf{R}^{k} .
$$

Similarly, if $\left|\Omega_{-} \backslash \Omega_{-}^{\circ}\right|=0$, then

$$
N^{-}(\lambda) \sim \int_{\Omega_{-}}(\lambda r(x))^{k / 2 m} \mu_{\mathcal{A}}^{\prime}(x) d x, \quad \text { as } \lambda \rightarrow-\infty .
$$

In particular, when $A=-\Delta$, we have

$$
\mu_{A}^{\prime}(x)=(2 \pi)^{-k} B_{k}
$$

where $B_{k}=\pi^{k / 2} / \Gamma(1+k / 2)$ denotes the volume of the unit ball in $\mathbf{R}^{k}$; since $N^{+}\left(\lambda_{n}^{+}\right)=n$, we then obtain an extension of Hermann Weyl's asymptotic formula (see Theorems 3.1 and 3.2):

$$
\left|\lambda_{n}^{ \pm}\right| \sim C_{k} n^{2 / k}\left\|r_{ \pm}\right\|_{L^{k / 2}(\Omega)}^{-1}, \quad \text { as } n \rightarrow \infty
$$

where $C_{k}=(2 \pi)^{2}\left(B_{k}\right)^{-2 / k}$ denotes Weyl's constant.

REMARKS. 1. Actually, (2.5) contains two equations: one involving the minus signs alone and a second one for the plus signs; we shall use this convenient sign convention throughout the paper. 
2. Note that the results for negative eigenvalues can be deduced directly from those for positive eigenvalues by changing $r$ into $-r$.

3. For simplicity, we write $\Omega_{ \pm}^{\circ}$ instead of $\left(\Omega_{ \pm}\right)^{\circ}$.

If, in addition, $\Omega$ is a bounded open set satisfying the segment property (for example, if $\partial \Omega$ is locally Lipschitz) and if $|\partial \Omega|=0$, the formulae (2.3) and (2.4) still hold for the Neumann problem (see Theorem 4.2).

When $r(x) \equiv 1, N^{-}(\lambda)=0$ and (2.3) is the usual formula (see, e.g., $[\mathbf{C H}$, Chapter VI, §4, pp. 429-445; Br; Ga; Kc; FlMe; Me; RS, Theorem XIII.78, p. 271 ; etc.]).

When $\Omega$ is unbounded, we assume that $A$ is an operator of "Schrödinger type", i.e., $A=\mathcal{L}+q$, where $\mathcal{L}$ satisfies $(2.1)$.

(2.6) Here, the "potential" $q$ is a nonnegative function on $\Omega$ such that $q^{-1}(x) r(x)$ $\rightarrow 0$ as $|x| \rightarrow+\infty$.

Under other suitable hypotheses, we show that the spectrum of $(\mathrm{P})$ is discrete. Moreover, we prove that (see Theorem 5.1 and Proposition 5.4)

$$
N^{ \pm}(\lambda) \sim \int_{\Omega_{ \pm} \cap \Omega_{\lambda}}(\lambda r(x)-q(x))^{k / 2 m} \mu_{\mathcal{L}}^{\prime}(x) d x, \quad \text { as } \lambda \rightarrow \pm \infty,
$$

if $\int_{\Omega}\left(r_{ \pm}\right)^{k / 2 m}=+\infty$, respectively; here, $\Omega_{\lambda}=\{x \in \Omega: \lambda r(x)>q(x)\}$ and $\mu_{\mathcal{L}}^{\prime}=\mu_{\mathcal{A}}^{\prime}$, by definition.

This estimate is well-known when $r(x) \equiv 1$. (See, e.g., [T, Chapter 17, §§8-14, pp. 174-185; Ro 2; Me; Fl; RS, Theorem XIII.81, p. 275; etc.].)

If $\int_{\Omega}\left(r_{ \pm}\right)^{k / 2 m}<+\infty$, then estimate (2.3) or (2.4) holds respectively as $\lambda \rightarrow \pm \infty$. (See Theorem 5.2 and Remark 1 following it as well as Proposition 5.4.)

We also establish lower bounds for the eigenvalues of the Dirichlet problem on a bounded domain $\Omega$; for instance, for the Laplacian and for $k \geq 3$, we have (see Theorem 3.3)

$$
\left|\lambda_{n}^{ \pm}\right| \geq \theta_{k} n^{2 / k}\left\|r_{ \pm}\right\|_{L^{k / 2}(\Omega)}^{-1}
$$

where $\theta_{k}$ is a constant depending only on $k$ which is explicitly known. In view of the extended Weyl formula (2.5), these lower bounds exhibit the correct "coupling constant behavior".

We refer the reader to Courant-Hilbert $[\mathbf{C H}$, Chapter VI], Reed-Simon [RS, Chapter XIII, $\S \S 1,15]$ or Weinberger [Wn, Chapter 3] for the Courant-Weyl method of proof of Weyl's formula. For the variational theory of elliptic boundary value problems, we point out Lions-Magenes [LM] and Agmon $[\mathbf{A g}]$. Finally, the basic properties of Sobolev spaces used in this paper can all be found in Adams [Ad].

3. An example: the Laplacian on an open set with finite volume. We now illustrate our methods for finding the asymptotic behavior of the eigenvalues, in the case of the Laplacian with Dirichlet or Neumann boundary conditions on an open set with finite volume or on a bounded open set, respectively (parts A and B). In part C, we obtain lower bounds for the eigenvalues of the corresponding Dirichlet problem on a bounded domain.

A. The Dirichlet problem. We consider the eigenvalue problem

$$
-\Delta u=\lambda r u, \quad \text { in } \Omega ; \quad u=0 \text { on } \partial \Omega .
$$


(3.1) $\Omega$ is an open subset of $\mathbf{R}^{k}$ with finite Lebesgue measure, satisfying (2.2) and such that $\left|\Omega_{+}\right| \Omega_{+}^{\circ} \mid=0$ and $\left|\Omega_{-}\right| \Omega_{-}^{\circ} \mid=0$.

The eigenvalues of the variational problem associated with $(\mathrm{E})$ are the complex numbers $\lambda$ such that there exists a nonzero $u \in H_{0}^{1}(\Omega)$ satisfying $-\Delta u=\lambda r u$ (in the distributional sense).

It is well known (see, e.g., [Ho; Pl; Wn, Chapter $3 ; \mathbf{M M}$; dF, Chapter I, $\S \S 1-2$; etc.]) that the eigenvalues $\lambda_{n}^{ \pm}$(when they exist) are characterized by the "max-min principle"

$$
\frac{1}{\lambda_{n}^{+}}=\max _{F_{n} \in \mathcal{F}_{n}} \min _{u \in F_{n}}\left\{\int_{\Omega} r|u|^{2}:\|\operatorname{grad} u\|_{L^{2}(\Omega)}^{2}=1\right\},
$$

where $\mathcal{F}_{n}$ denotes the set of $n$-dimensional subspaces of $H_{0}^{1}(\Omega)$; an analogous formula holds for $\lambda_{n}^{-}$.

The hypothesis $\left|\Omega_{ \pm}\right|>0$ implies that $\lambda_{n}^{ \pm}$exists for all $n \geq 1$ and $\lambda_{n}^{ \pm} \rightarrow \pm \infty$ as $n \rightarrow \infty$. The precise asymptotic behavior is given by

THEOREM 3.1. Under assumptions (2.2) and (3.1), estimates (2.3)-(2.5) hold.

If $r$ is positive in $\Omega$, we have $N^{-}(\lambda)=0$ (actually, this happens if and only if $\left.\left|\Omega_{-}\right|=0\right)$. Formulae $(2.3)$ and $(2.5)$ - which are clearly equivalent-are classical when $r$ is positive and continuous (see, e.g., $[\mathbf{C H}$, Theorem 14, p. 435; RS, Theorem XIII.78, p. 271; Me, Theorem 5.12, p. 188; etc.]).

We shall need two lemmas in order to derive Theorem 3.1. The following result is essentially known and for $k \geq 3$, for instance, can be obtained by combining [RS, Theorem XIII.80, p. 274] and [LiY, Step (iv), pp. 317-318]. (For the case $k=1$, see [GoK, Theorem 8.1, p. 309].)

LEMMA 3.1. When $r$ is positive in $L^{p}(\Omega)$ with $p>k / 2$ if $k \geq 3$ and $p=1$ if $k<3,(2.3)$ and (2.5) hold.

In the following, we shall denote by $\lambda_{n}^{+}(r, \Omega)$ the $n$th nonnegative eigenvalue of (E) to indicate the dependence on $r$ and $\Omega$.

LEMMA 3.2 (MONOTONICITY PRINCIPLES). $\lambda_{n}^{+}(r, \Omega)$ does not increase when $r$ or $\Omega$ increases.

REMARKS. 1. Here and thereafter, we adopt the following convention: whenever we make use of an eigenvalue $\lambda_{n}^{+}$, we implicitly assume that it exists (equivalently, that the right-hand side of (3.2) - or of its counterpart-is finite).

2. The monotonicity with respect to $\Omega$ is only valid for Dirichlet problems.

Lemma 3.2 is a simple consequence of (3.2) and is well-known when $r$ is positive (see, e.g., [CH, Theorem 3, p. 409 and Theorem 7, p. 411; RS, Proposition 4, p. 270 and Wn, Theorem 7.1, p. 58, Example 7.2, p. 60 and Theorem 8.1, p. 62].

PROOF OF THEOREM 3.1. We follow the method used in [La 1, La 2]: the idea consists in finding lower and upper bounds for $\lambda_{n}^{+}$having the same asymptotics. With this aim and the help of the monotonicity principles, we reduce the problem to the study of positive weights.

It clearly suffices to establish $(2.5)$ for $\lambda_{n}^{+}$since $\lambda_{n}^{-}(r, \Omega)=-\lambda_{n}^{+}(-r, \Omega)$ and $r_{-}=(-r)_{+}$.

Fix $\delta>0$. By Lemma 3.2, we have

$$
\lambda_{n}^{+}\left(r_{+}+\delta, \Omega\right) \leq \lambda_{n}^{+}(r, \Omega) \leq \lambda_{n}^{+}\left(r, \Omega_{+}^{\circ}\right)
$$


this holds since $r \leq r_{+}+\delta$ and $\Omega_{+}^{\circ}$ is an open subset of $\Omega$ of positive measure, by (2.2) and (3.1).

By Lemma 3.1 applied to the positive weights $r_{+}+\delta$ in $\Omega$ and $r$ in $\Omega_{+}^{\circ}$, we see that

$$
\lim _{n \rightarrow \infty} n^{-2 / k} \lambda_{n}^{+}\left(r_{+}+\delta, \Omega\right)=C_{k}\left\|r_{+}+\delta\right\|_{L^{k / 2}(\Omega)}^{-1}
$$

and

$$
\lim _{n \rightarrow \infty} n^{-2 / k} \lambda_{n}^{+}\left(r, \Omega_{+}^{\circ}\right)=C_{k}\|r\|_{L^{k / 2}\left(\Omega_{+}^{\circ}\right)}^{-1} .
$$

We infer from equations (3.3) to (3.5) that

$$
\begin{aligned}
C_{k}\left\|r_{+}+\delta\right\|_{L^{k / 2}(\Omega)}^{-1} & \leq \varliminf_{n \rightarrow \infty} n^{-2 / k} \lambda_{n}^{+}(r, \Omega) \\
& \leq \varlimsup_{n \rightarrow \infty} n^{-2 / k} \lambda_{n}^{+}(r, \Omega) \leq C_{k}\|r\|_{L^{k / 2}\left(\Omega_{+}^{\circ}\right)}^{-1}
\end{aligned}
$$

Now, it follows from the Lebesgue dominated convergence theorem that $\left\|r_{+}+\delta\right\|_{L^{k / 2}(\Omega)}$ tends to $\|r\|_{L^{k / 2}\left(\Omega_{+}^{\circ}\right)}$ as $\delta$ tends to 0 ; moreover, by (3.1),

$$
\int_{\Omega_{+}^{\circ}} r^{k / 2}=\int_{\Omega_{+}} r^{k / 2}=\int_{\Omega}\left(r_{+}\right)^{k / 2} .
$$

[Note that by omitting the zero set of $r$, one does not alter the value of the latter integral.]

Consequently, we obtain (2.5) by letting $\delta$ tend to zero in (3.6).

REMARKS. 1. When $r$ is continuous, $\Omega_{+}$is open and Theorem 3.1 holds for an arbitrary open subset $\Omega$ of $\mathbf{R}^{k}$ with finite Lebesgue measure (in particular, for any bounded open set).

2. When $\Omega_{+}$is a "Jordan contended set" (roughly speaking, if $\Omega_{+}$is bounded and well approximated from within and without by a finite union of cubes-see, e.g., [LoS, Chapter 8, $\S \S 6-7$; RS, p. 271; Pe, Chapter 2, $\S \S 11-12])$, we have $\left|\Omega_{+}\right| \Omega_{+}^{\circ} \mid=0$ since then, by [LoS, Proposition 6.1, p. 332], $\left|\partial\left(\Omega_{+}\right)\right|=0$; this was the assumption of [La 1, Theorem 1, p. 266].

B. The Neumann problem. We suppose that

(3.7) $\Omega$ is a bounded open set satisfying the segment property (see $[\mathbf{A g}$, Definition 2.1 , p. 11]) such that $|\partial \Omega|=0,\left|\Omega_{+}\right| \Omega_{+}^{\circ} \mid=0$ and $\left|\Omega_{-}\right| \Omega_{-}^{\circ} \mid=0$.

We consider the eigenvalue problem

$$
-\Delta u=\lambda r u \quad \text { in } \Omega ; \quad \partial u / \partial n=0 \quad \text { on } \partial \Omega
$$

where $\partial / \partial n$ denotes the normal derivative.

The scalar $\lambda$ is said to be an eigenvalue of the variational problem associated with $\left(\mathrm{E}^{\prime}\right)$ if $-\Delta u=\lambda r u$ for some nonzero $u \in H^{1}(\Omega)$.

The corresponding nonnegative eigenvalues $\Lambda_{n}^{+}(r, \Omega)$ are, in this case, given by (3.2), where $H_{0}^{1}(\Omega)$ is replaced by $H^{1}(\Omega)$.

Since $H_{0}^{1}(\Omega) \subset H^{1}(\Omega)$, it follows from the "max-min principle" as in $[\mathbf{C H}$, Theorem 5, p. 410; RS, Proposition 4, p. 270 or Wn, Theorem 7.1, p. 58 and Example 7.4, pp. 61-62] that

$$
\Lambda_{n}^{+}(r, \Omega) \leq \lambda_{n}^{+}(r, \Omega)
$$


and, as above, the eigenvalues are monotone with respect to the weight; therefore, for $\delta>0$, we have

$$
\Lambda_{n}^{+}\left(r_{+}+\delta, \Omega\right) \leq \Lambda_{n}^{+}(r, \Omega) \leq \lambda_{n}^{+}(r, \Omega) .
$$

As for the Dirichlet problem, using an extension of (2.3) or (2.5) when $\Omega$ satisfies (3.7) and the weight is positive [FIMe; Me, Theorem 5.12, p. 188], we obtain

THEOREM 3.2. Under assumptions (2.1), (2.2) and (3.7), estimates (2.3)-(2.5) hold.

Next, we comment on possible extensions or interpretations of the above results:

REMARKS. 1. The hypothesis concerning the segment property in Theorem 3.2 can be weakened as in [Me, Condition $\left(\mathrm{C}^{\prime}\right)$, p. 156].

2. It is noteworthy that no assumption has been made about $\Omega_{0}=\{x \in \Omega: r(x)=$ $0\}$ in order to obtain Theorems 3.1 and 3.2.

3 . It is easy to derive from Theorems 3.1 and 3.2 a corresponding result for mixed Dirichlet-Neumann boundary conditions; naturally, the asymptotic distribution of the eigenvalues remains unchanged in this case.

4. The conclusions of Theorems 3.1 or 3.2 can be interpreted as follows: the positive (resp., negative) eigenvalues of $(\mathrm{E})$ or $\left(\mathrm{E}^{\prime}\right)$ have the same asymptotic behavior as the eigenvalues - in the usual sense - of the elliptic operator $-\Delta / r(x)$ [resp., $+\Delta / r(x)]$, defined in $\Omega_{+}^{\circ}$ (resp., $\Omega_{-}^{\circ}$ ) for the corresponding boundary conditions. Here, we use implicitly the fact that $\left\|r_{ \pm}\right\|_{L^{k / 2}(\Omega)}=\|r\|_{L^{k / 2}\left(\Omega_{ \pm}^{\circ}\right)}$.

[The preceding remarks apply, with obvious changes, to the general elliptic boundary value problems studied in $§ 4$.B.]

5. Physically, when $r$ is positive, the eigenvalues of $(\mathrm{E})$ represent the natural frequencies of a vibrating membrane fixed along its boundary $\partial \Omega$ and of mass density equal to $r$-as well as of tension unity. (See, e.g., [CH, Chapters V and VI or Kc].) Therefore, in the case when $r$ changes sign, we may interpret Theorem 3.1 by saying that the large values of $\lambda_{n}^{+}$(resp., $\lambda_{n}^{-}$) are determined by the "positive mass" (resp., "negative mass") distribution of the "membrane".

EXAMPLE 3.1. We now consider a simple example of discontinuous weight function. Naturally, our assumptions would allow us to treat much more singular weights.

Let $\Omega$ be an open subset of $\mathbf{R}^{k}$ with finite measure $|\Omega|$. Let $\Omega_{+}$and $\Omega_{-}$be two disjoint measurable subsets of $\Omega$ of positive measure such that $\left|\Omega_{+}\right| \Omega_{+}^{\circ} \mid=0$ and $\left|\Omega_{-} \backslash \Omega_{-}^{\circ}\right|=0$. We define

$$
r(x)= \begin{cases}-1 & \text { if } x \in \Omega_{-} \\ 0 & \text { if } x \in \Omega \backslash\left(\Omega_{-} \cup \Omega_{+}\right), \\ +1 & \text { if } x \in \Omega_{+}\end{cases}
$$

We then deduce from Theorem 3.1 that, for the Dirichlet problem, we have

$$
\text { COROLlaRY 3.1. } \lambda_{n}^{ \pm} \sim C_{k}\left(n /\left|\Omega_{ \pm}\right|\right)^{2 / k} \text {, as } n \rightarrow \infty \text {. }
$$

The same estimate holds for the Neumann problem under the additional assumptions that $|\partial \Omega|=0$ and $\Omega$ is bounded and satisfies the segment property.

REMARK. The geometric features of the above example suggest the following natural extension of the well-known isospectral problem (see, e.g., [Kc and Y, pp. 
23-24]): let $\Omega^{1}, \Omega_{ \pm}^{1}, r_{1}$ and $\Omega^{2}, \Omega_{ \pm}^{2}, r_{2}$, be defined as in Example 3.1. Assume that the problem (E) [or $\left.\left(\mathrm{E}^{\prime}\right)\right]$ has the same set of eigenvalues, counting multiplicities, in $\Omega_{1}$ and $\Omega_{2}$. Under which (generic) conditions does there exist an isometry which sends $\Omega^{1}, \Omega_{+}^{1}$ and $\Omega_{-}^{1}$ onto, respectively, $\Omega^{2}, \Omega_{+}^{2}$ and $\Omega_{-}^{2}$ ? In other words, to what extent does the spectrum determine the sign of the weight?

C. Lower bounds of the eigenvalues. We obtain here lower bounds (resp., upper) bounds for the eigenvalues $\lambda_{n}^{+}$(resp., $\lambda_{n}^{-}$) of the Dirichlet problem (E) studied in part $\mathrm{A}$. These bounds are valid for all $n$ and compatible with the asymptotic behavior obtained in Theorem 3.1.

THEOREM 3.3. Assume that $\Omega$ is a bounded domain of $\mathbf{R}^{k}$ with $C^{2}$ boundary $\partial \Omega$ and suppose that $k \geq 3$. Then, if $\left|\Omega_{ \pm}\right|>0$, respectively, we have for the Dirichlet problem (E) in $\Omega$

$$
\left|\lambda_{n}^{ \pm}\right| \geq \theta_{k} n^{2 / k}\left\|r_{ \pm}\right\|_{L^{k / 2}(\Omega)}^{-1}, \quad \forall n \geq 1,
$$

where $\theta_{k}=(k(k-2) / 4 e)\left(\omega_{k-1}\right)^{2 / k}, e=\exp (1)$ and $\omega_{k-1}=k B_{k}$ denotes the area of the unit sphere in $\mathbf{R}^{k}$.

ProOF. It clearly suffices to find a lower bound for $\lambda_{n}^{+}$when $\left|\Omega_{+}\right|>0$. Fix $n \geq 1$ and $\delta>0$. Since $r \leq r_{+}+\delta$, we derive from Lemma 3.2 that

$$
\lambda_{n}^{+}(r, \Omega) \geq \lambda_{n}^{+}\left(r_{+}+\delta, \Omega\right) .
$$

By [LiY, Theorem 2, p. 314] applied to the positive weight $r_{+}+\delta$, we have

$$
\lambda_{n}^{+}\left(r_{+}+\delta, \Omega\right) \geq \theta_{k} n^{2 / k}\left\|r_{+}+\delta\right\|_{L^{k / 2}(\Omega)}^{-1} .
$$

Combining (3.11) and (3.12), we now obtain (3.10) by letting $\delta \rightarrow 0$ and applying the dominated convergence theorem.

Since $N^{+}\left(\lambda_{n}^{+}\right)=n$, we deduce

COROllary 3.2 .

$$
\left(\frac{k(k-2)}{4 e}\right)^{k / 2} \omega_{k-1} N^{ \pm}(\lambda) \leq \lambda^{k / 2}\left(\int_{\Omega}\left(r_{ \pm}\right)^{k / 2}\right), \quad \forall \lambda>0 .
$$

We have just used a remarkable result of $\mathrm{Li}$ and Yau [LiY, Theorem 2, p. 314] which improved significantly the existing upper bounds for the number of "bound states" of the Schrödinger operator in $\mathbf{R}^{3}$. (See [LiY, p. 311 and Lb, p. 243] for references to previous works on this subject.) Note, however, that the method of $\mathrm{Li}$ and Yau does not seem a priori to apply to indefinite weights.

REMARKS. 1. The assumption that $\left|\Omega_{ \pm}\right| \Omega_{ \pm}^{\circ} \mid=0$ is not needed for Theorem 3.3 to hold.

2. We note that upper bounds for $\left|\lambda_{n}^{ \pm}\right|$can be deduced similarly from the known upper bounds for the eigenvalues of Dirichlet problems with positive weights. Indeed, by $(3.5),\left|\lambda_{n}^{ \pm}(r, \Omega)\right| \leq\left|\lambda_{n}^{ \pm}\left(r, \Omega_{ \pm}^{\circ}\right)\right|$.

3. As is the case with the result of $\mathrm{Li}$ and $\mathrm{Yau}$, Theorem 3.3 extends to a compact Riemannian manifold with $C^{2}$ boundary; $\theta_{k}$ must then be replaced by a constant depending on the Sobolev constant of the manifold.

4. Theorem 3.3 also extends to general selfadjoint elliptic operators of order 2; in this case, the new constant occurring in (3.10) depends on $\theta_{k}$ and the constant 
of uniform ellipticity of the operator. This is easily seen by using a monotonicity argument (based on Lemma 4.2) and Theorem 3.3.

5. It may be worth pointing out that, for $k \geq 3$, Theorem 3.3 provides us with a new proof of the fact that $\lambda_{n}^{ \pm}$exists for all $n \geq 1$ and $\lambda_{n}^{ \pm} \rightarrow \pm \infty$ when $\left|\Omega_{ \pm}\right|>0$. (Compare with [MM, Proof of Proposition 3, p. 290 or dF, Proof of Proposition 1.11 , p. 43].)

4. Elliptic boundary value problems with bounded coefficients. We extend the results of $\S \S 3$.A and 3.B to selfadjoint elliptic operators of order $2 \mathrm{~m}$.

A. Abstract theory. In order to obtain results on more general boundary value problems, we briefly explain the abstract theory (see, e.g., [Wn, Chapter 3 and dF, Chapter I]). It will later be applied to operators with bounded ( $\S 4$.B) as well as unbounded ( $\$ 5$ ) coefficients (that is, in the latter case, Schrödinger operators).

(4.1) Let $H$ be a real or complex Hilbert space.

(4.2) Let $(V, H, a)$ be a variational triple: $V$ is a dense subspace of $H$ with continuous imbedding and $a$ is a hermitian, bounded and coercive form defined on $V($ see $[\mathbf{L M}])$.

(4.3) $b$ is a hermitian form on $V$ such that, for some positive constant $\delta$,

$$
|b(u, u)| \leq \delta a(u, u), \quad \forall u \in V .
$$

We consider the following variational eigenvalue problem

$$
a(u, v)=\lambda b(u, v), \quad \forall v \in V
$$

here, $\lambda$ is an eigenvalue of $(\mathrm{Q})$ [or is in the spectrum of $(\mathrm{Q})$ ] if there exists a nonzero $u \in V$ such that the last equation holds.

REMARK. In the example of $\S 3$, we consider $H=L^{2}(\Omega), a(u, u)=\|u\|_{H^{1}(\Omega)}^{2}$, $b(u, u)=\int_{\Omega} r|u|^{2}$ and $V=H_{0}^{1}(\Omega)$ [resp., $V=H^{1}(\Omega)$ ] for the Dirichlet [resp., Neumann] boundary value problem on an open set $\Omega$ with finite volume (resp., on a bounded open set $\Omega$ ). In this manner, Theorems 3.1 and 3.2 will become corollaries of Theorems 4.1 and 4.2 , respectively.

By (4.2), $a$ induces in $V$ an inner product equivalent to the original one; by the Riesz representation theorem, we define a bounded selfadjoint operator $T$ from $(V, a(\cdot, \cdot))$ to itself by

$$
b(u, v)=a(T u, v), \quad \forall(u, v) \in V \times V .
$$

It follows that $\lambda$ is an eigenvalue of $(Q)$ if and only if $1 / \lambda$ is an eigenvalue of $T: T u=(1 / \lambda) u$, for some nonzero $u \in V$.

(4.5) We now assume that $T$ is compact. This is the case in all the problems studied here; it holds in particular if $V$ is compactly embedded in $H$ and $b$ is continuous on $H$.

It follows from the classical spectral theory of compact selfadjoint operators in Hilbert spaces that the spectrum of $(\mathrm{Q})$ consists of a double sequence

$$
\cdots \leq \lambda_{n+1}^{-} \leq \lambda_{n}^{-} \leq \cdots \leq \lambda_{1}^{-}<(0) \leq \lambda_{1}^{+} \leq \cdots \leq \lambda_{n}^{+} \leq \lambda_{n+1}^{+} \leq \cdots
$$

(each eigenvalue has finite multiplicity and is repeated accordingly).

When it exists, $\lambda_{n}^{ \pm}$- which we write $\lambda_{n}^{ \pm}(a, V)$ or $\lambda_{n}^{ \pm}(b, V)$ if necessary-is given by the "max-min principle"

$$
\frac{1}{\lambda_{n}^{+}}=\max _{F_{n} \in \mathcal{F}_{n}} \min _{u \in F_{n}}\{b(u, u): a(u, u)=1\},
$$


where $\mathcal{F}_{n}$ is the set of $n$-dimensional subspaces of $V$. A similar formula holds for $\lambda_{n}^{-}$since $\lambda_{n}^{-}(V,-b)=-\lambda_{n}^{+}(V, b)$.

Just as before, we deduce from (4.6) the following abstract monotonicity principles:

LEMMA 4.1. If $b_{1}, b_{2}$ are two hermitian forms satisfying (4.3) and such that $b_{1}(u, u) \leq b_{2}(u, u)$ for all $u \in V$, then $\lambda_{n}^{+}\left(b_{1}, V\right) \geq \lambda_{n}^{+}\left(b_{2}, V\right)$.

LEMMA 4.2. Let $a_{1}, a_{2}$ be coercive forms satisfying (4.2) and such that $a_{1}(u, u)$ $\geq a_{2}(u, u)$ for all $u \in V$; then $\lambda_{n}^{+}\left(a_{1}, V\right) \geq \lambda_{n}^{+}\left(a_{2}, V\right)$.

LEMMA 4.3. If $\left(V_{1}, H, a\right)$ and $\left(V_{2}, H, a\right)$ are two "variational triples" such that $V_{1} \subset V_{2}$, then $\lambda_{n}^{+}\left(b, V_{1}\right) \geq \lambda_{n}^{+}\left(b, V_{2}\right)$.

B. Operators of order $2 m$. In the following, we suppose that $\Omega$ is a nonempty open subset of $\mathbf{R}^{k}$ and that $r$ and $\Omega$ satisfy (2.2); further the operator $A=$ $\sum_{|\alpha| \leq m,|\beta| \leq m} D^{\alpha}\left(a_{\alpha \beta} D^{\beta}\right)$ fulfills (2.1).

We first consider the Dirichlet boundary value problem: $A u=\lambda r u, u \in H_{0}^{m}(\Omega)$. 0 .

(4.7) We assume that $\Omega$ has finite volume and that $\left|\Omega_{+}\right| \Omega_{+}^{\circ} \mid=0$ and $\left|\Omega_{-}\right| \Omega_{-}^{\circ} \mid=$

We can now apply the above abstract theory with $H=L^{2}(\Omega), V=H_{0}^{m}(\Omega)$ and

$$
a(u, v)=\int_{\Omega} \sum_{\substack{|\alpha| \leq m \\|\beta| \leq m}} a_{\alpha \beta} D^{\alpha} u \overline{D^{\beta} v}
$$

It follows from standard results on Sobolev spaces and from the uniform ellipticity of $\mathcal{A}$ (i.e., $\mathcal{A}^{\prime}(x, \xi) \geq c|\xi|^{2 m}$, for all $\xi \in \mathbf{R}^{k}$ and some $c>0$ ) that $a$ satisfies (4.2).

By (2.2) and the Sobolev imbedding theorem [Ad, Theorem 5.4, p. 97], we see that $b(u, v)=\int_{\Omega} r u \bar{v}$ satisfies (4.3).

When $k>2 m$, we also have, by using the Sobolev and the Hölder inequalities that, for $u, v \in V$

$$
\left|\int_{\Omega} r u \bar{v}\right| \leq\|r\|_{L^{p}(\Omega)}\|u\|_{L^{s}(\Omega)}\|v\|_{L^{2^{*}}(\Omega)}
$$

where $2^{*}=2 k /(k-2 m)$ and $1 / p+1 / s+1 / 2^{*}=1$.

Therefore, (4.5) follows from the Rellich-Kondrachov theorem [Ad, Theorem 6.2 , p. 144]. Similar results are obtained when $k \leq 2 m$ by use of [Ad, Theorem 5.4, Cases B and C, p. 97 and Theorem 6.2, equations (4) and (6), p. 144]. Much like in [dF, Proof of Proposition 1.11, p. 43], one then shows that the assumption $\left|\Omega_{ \pm}\right|>0$ implies that $\left|\lambda_{n}^{ \pm}\right| \rightarrow+\infty$ as $n \rightarrow \infty$.

We now state

THEOREM 4.1. Under hypotheses (2.1), (2.2) and (4.7), estimates (2.3) and (2.4) hold and

$$
\left|\lambda_{n}^{ \pm}\right| \sim n^{2 m / k}\left(\int_{\Omega_{ \pm}} r^{k / 2 m}(x) \mu_{\mathcal{A}}^{\prime}(x) d x\right)^{-2 m / k}, \quad \text { as } n \rightarrow \infty .
$$

PROOF. Estimate (2.3) holds when $r$ is continuous and bounded away from zero (see [Me, Theorem 5.12, p. 188]); hence, for $r$ nonnegative, we obtain the same 
result by replacing $r$ by $r+\delta$ and letting $\delta \rightarrow 0$. In light of Lemmas 4.1 and 4.3, we then obtain (4.8) and (2.3) exactly as in the proof of Theorem 3.1. Note that

$$
\int_{\Omega_{ \pm}} r^{k / 2 m} \mu_{\mathcal{A}}^{\prime}=\int_{\Omega_{ \pm}^{\circ}} r^{k / 2 m} \mu_{\mathcal{A}}^{\prime}=\int_{\Omega}\left(r_{ \pm}\right)^{k / 2 m} \mu_{\mathcal{A}}^{\prime} .
$$

Likewise, we have

THEOREM 4.2. For the Neumann problem, if $r, \Omega$ and $A$ satisfy (2.1), (2.2) and (3.7), then (2.3), (2.4) and (4.8) hold.

ProOF. We now consider $V=H^{m}(\Omega)$; we use, in particular, Lemma 4.3 with $V_{1}=H_{0}^{m}(\Omega)$ and $V_{2}=H^{m}(\Omega)$; and the proof goes through as in Theorems 3.2 and 4.1 .

5. The Schrödinger operator. In this section, we give sufficient conditions under which a positive operator of "Schrödinger type" and with an indefinite weight has discrete "spectrum" in an unbounded open set $\Omega \subset \mathbf{R}^{k}$ and we then determine the asymptotic distribution of its eigenvalues. We combine, in particular, the results and methods of $\S 4$ and previous works [Ro 2; Ro 3; Fl; etc.] on Schrödinger operators with positive weights.

DEFINITION 5.1. Let $f$ be a continuous function defined on an open subset $\omega$ of $\mathbf{R}^{k}$. We say that $f$ satisfies $(\varepsilon)$ if there exists a positive number $\varepsilon_{0}$ such that $f$ can be continuously extended to $\tilde{\omega}=\left\{x \in \mathbf{R}^{k}: \operatorname{dist}(x, \omega)<\varepsilon_{0}\right\}$ and, for all $\varepsilon \in\left(0, \varepsilon_{0}\right)$, there exists $\eta>0$ such that, for all $(x, y) \in \tilde{\omega} \times \tilde{\omega}$ with $|x-y| \leq \eta$, we have $|f(x)-f(y)| \leq \varepsilon|f(x)|$.

REMARKS. 1. For instance, $f(x)=\left(1+|x|^{2}\right)^{\sigma}$, with $\sigma \geq 1$, satisfies $(\varepsilon)$.

2. If a positive function $f$ satisfies $(\varepsilon)$, so does its inverse $1 / f$.

In the following, we set $\Omega_{\rho}^{\prime}=\{x \in \Omega:|x|>\rho\}$ for $\rho>0$.

(5.1) Let $q$ be a nonnegative function in $L_{\text {loc }}^{k / 2 m}(\Omega)$, tending to $+\infty$ at infinity and satisfying $(\varepsilon)$ on $\Omega_{R_{1}}^{\prime} \cap \Omega_{+}$, for some $R_{1}>0$.

(5.2) Let $r$ be a function defined on $\Omega$ such that $q^{-1} r$ tends to 0 at infinity. Moreover, we suppose that

(a) Assumption (2.2) holds and $\left|\Omega_{+} \backslash \Omega_{+}^{\circ}\right|=0$.

(b) The restriction of $r$ to $\Omega_{R_{1}}^{\prime} \cap \Omega_{+}$satisfies $(\varepsilon)$.

(c) $\int_{\Omega}\left(r_{+}\right)^{k / 2 m}=+\infty$.

(Note that $r$ is allowed to change sign anywhere in $\Omega$.)

REMARK. (5.2) holds, for example, if $\Omega=\mathbf{R}^{k}$ with $r(x)=-1$ if $|x| \leq 1$ and $r(x)=+1$ if $|x|>1$.

(5.3) Let $\mathcal{L}$ be a formally selfadjoint, uniformly elliptic operator of order $2 m$ defined on $\Omega$ and verifying (2.1):

$$
\mathcal{L}=\sum_{\substack{|\alpha| \leq m \\|\beta| \leq m}} D^{\alpha}\left(a_{\alpha \beta} D^{\beta}\right)
$$

we suppose that for all $|\alpha|=|\beta|=m, a_{\alpha \beta}$ satisfies $(\varepsilon)$ on $\Omega_{R_{1}}^{\prime}$; in addition, we assume that there exists $d>0$ such that for all open subsets $\omega$ of $\Omega$,

$$
\int_{\omega} \sum_{|\alpha|=|\beta|=m} a_{\alpha \beta} D^{\alpha} u \overline{D^{\beta} u} \geq d\|u\|_{H^{m}(\omega)}^{2}, \quad \forall u \in H^{m}(\omega) .
$$


DEFINITION 5.2. If $\omega$ is an open subset of $\mathbf{R}^{k}, V_{q}^{0}(\omega)$ denotes the completion of $C_{0}^{\infty}(\omega)$ with respect to the Hilbert norm

$$
\|u\|_{V_{q}(\omega)}=\left(\|u\|_{H^{m}(\omega)}^{2}+\int_{\omega} q|u|^{2}\right)^{1 / 2} .
$$

The space $V_{q}^{1}(\omega)$ is the set of restrictions to $\omega$ of elements of $V_{q}^{0}(\Omega)$.

We study the variational eigenvalue problem

(S) $\quad A u=(\mathcal{L}+q) u=\lambda r u$, in $\Omega$, with Dirichlet boundary conditions.

In other words, $\lambda$ is an eigenvalue of $(\mathrm{S})$ if there exists a nonzero $u$ in $V_{q}^{0}(\Omega)$ such that the above equation holds in the distributional sense.

We now apply the abstract theory developed in $\S 4$.A; here, $H=L^{2}(\Omega)$ is equipped with its usual inner product $(),, V=V_{q}^{0}(\Omega), b(u, u)=(r u, u)$ and $a(u, u)=(\mathcal{L} u, u)+(q u, u)$, for $u \in V$.

It follows from (5.1) and (5.3) that $a$ is coercive on $V_{q}^{0}(\Omega)$; further, the fact that $q$ tends to infinity at infinity implies that the imbedding of $V_{q}^{0}(\Omega)$ into $L^{2}(\Omega)$ is compact. Moreover, (4.5) holds because, for $\rho>0$,

$$
\left.\left|\int_{\Omega_{\rho}^{\prime}} r\right| u\right|^{2} \mid \leq\left(\sup _{x \in \Omega_{\rho}^{\prime}}\left|\frac{r}{q}\right|\right)\left(\int_{\Omega_{\rho}^{\prime}} q|u|^{2}\right) \leq \delta(\rho)\|u\|_{V_{q}\left(\Omega_{\rho}^{\prime}\right)}^{2},
$$

where $\delta(\rho)$ tends to zero as $\rho \rightarrow+\infty$; this follows since, by (5.2), $r / q$ tends to zero at infinity.

Consequently, the spectrum of (S) is discrete; moreover, the eigenvalues of (S) are characterized by the "max-min" principle" (4.6).

Let $\lambda_{n}^{+}(r, \omega)$ [resp., $\left.\Lambda_{n}^{+}(r, \omega)\right]$ denote the nonnegative eigenvalues of the variational Dirichlet [resp., Neumann] boundary value problem

$$
\left.A u=\lambda r u, \quad \text { in } \omega \subset \Omega ; \quad u \in V_{q}^{0}(\omega) \quad \text { [resp., } u \in V_{q}^{1}(\omega)\right] .
$$

Set $N_{0}^{+}(\lambda ; r, \omega, \mathcal{A})=\sum_{\lambda_{n}^{+}(r, \omega)<\lambda} 1$ and $N_{1}^{+}(\lambda ; r, \omega, \mathcal{A})=\sum_{\Lambda_{n}^{+}(r, \omega)<\lambda} 1$; one indicates in this way the dependence on the weight function $r$, the open set $\omega$ and the operator $A$.

Note that

$$
N_{0}^{+}(\lambda ; r, \Omega, A)=N^{+}(\lambda),
$$

where $N^{+}(\lambda)$ stands for the number of nonnegative eigenvalues of (S) less than $\lambda>0$.

We shall use the subsequent lemma, which is derived from Lemma 4.3.

LEMMA 5.1. If $\omega_{1}$ and $\omega_{2}$ are two disjoint open sets in $\omega \subset \Omega$ such that $\bar{\omega}_{1} \cup \bar{\omega}_{2}=\bar{\omega}$, then

$$
\begin{aligned}
N_{0}^{+}\left(\lambda ; r, \omega_{1}, \mathcal{A}\right)+N_{0}^{+}\left(\lambda ; r, \omega_{2}, \mathcal{A}\right) & \leq N_{0}^{+}(\lambda ; r, \omega, \mathcal{A}) \leq N_{1}^{+}(\lambda ; r, \omega, \mathcal{A}) \\
& \leq N_{1}^{+}\left(\lambda ; r, \omega_{1}, \mathcal{A}\right)+N_{1}^{+}\left(\lambda ; r, \omega_{2}, \mathcal{A}\right) .
\end{aligned}
$$

This is due in particular to the inclusions

$$
V_{q}^{0}\left(\omega_{1}\right) \oplus V_{q}^{0}\left(\omega_{2}\right) \subset V_{q}^{0}(\omega) \quad \text { and } \quad V_{q}^{1}(\omega) \subset V_{q}^{1}\left(\omega_{1}\right) \oplus V_{q}^{1}\left(\omega_{2}\right)
$$


Let us consider a covering of $\mathbf{R}^{k}$ by a countable family of disjoint open cubes $\left\{Q_{\zeta}\right\}_{\zeta \in \mathbf{Z}^{k}}$ with center $x_{\zeta}$ and sides of length $\eta$ :

$$
\mathbf{R}^{k}=\bigcup_{\varsigma \in \mathbf{Z}^{k}} \bar{Q}_{\zeta} .
$$

Let $R>R_{1}$. Set $B=\{x \in \Omega:|x|<R\}$ and $G=\Omega_{R}^{\prime}=\{x \in \Omega:|x|>R\}$; write

$$
G_{+}=\{x \in G: r(x)>0\} \quad \text { and } G_{-}=\{x \in G: r(x)<0\} .
$$

Fix $\lambda>0$; let $G_{\lambda}=\{x \in G: \lambda r(x)-q(x)>0\}$.

We note that $G_{+}$and $G_{\lambda}$ are open; moreover, we have

$$
G_{\lambda} \subset G_{+} \text {and } \operatorname{dist}\left(G_{\lambda}, G \backslash G_{+}\right)>0
$$

this follows since $r \geq \gamma / \lambda$ on $G_{\lambda}$ and $r, q$ are continuous on $G_{+}$; here and thereafter, we choose $R_{1}$ (and $R>R_{1}$ ) so large that $q \geq \gamma$ on $\Omega_{R_{1}}^{\prime}$ for some positive constant $\gamma$; this is possible in view of (5.1). Since, by (5.2), $G_{\lambda}$ is bounded, the sets $I$ and $J$ that we now introduce are finite.

Put $I=\left\{\varsigma \in \mathbf{Z}^{k}: \bar{Q}_{\zeta} \subset G_{\lambda}\right\}$ and $J=\left\{\varsigma \in \mathbf{Z}^{k}: \bar{Q}_{\zeta} \cap \bar{G}_{\lambda} \neq \varnothing\right\}$. We choose $\eta$ so small that $\left(\bigcup_{\varsigma \in J} \bar{Q}_{\zeta}\right) \subset G_{+}$.

We further make some technical assumptions on $r, q$ and $G_{\lambda}$ (recall that $r$ is positive on $G_{\lambda}$ ):

$$
\lim _{\eta \rightarrow 0}\left(\sum_{\zeta \in J \backslash I}\left(r_{\zeta}\right)^{k / 2 m}\right)\left(\sum_{\zeta \in I}\left(r_{\zeta}\right)^{k / 2 m}\right)^{-1}=0,
$$

where $r_{\zeta}=r\left(x_{\zeta}\right)$ for $\zeta \in \mathbf{Z}^{k}$.

(5.6) Finally, we suppose that there exist $\lambda^{\prime}>0$ and $c>0$ such that $G_{\lambda}$ is Jordan contended (see [LoS, Chapter 8]) and $\left[G_{\lambda}\right] \leq c\left[G_{\lambda / 2}\right], \forall \lambda \geq \lambda^{\prime}$, where $\left[G_{\lambda}\right]=\int_{G_{\lambda}} r^{k / 2 m}$. Intuitively, (5.5) means that $\partial G_{\lambda}$ is of zero measure with respect to $r(x) d x$; moreover, hypothesis (5.6) is of Tauberian type.

We can now state

THEOREM 5.1. Under hypotheses (5.1)-(5.3) and (5.5)-(5.6), the spectrum of (S) is discrete and estimate (2.7) holds:

$$
N^{+}(\lambda) \sim \int_{\Omega_{+}}\left[(\lambda r-q)_{+}\right]^{k / 2 m} \mu_{\mathcal{L}}^{\prime}, \quad \text { as } \lambda \rightarrow+\infty
$$

where $\Omega_{+}=\{x \in \Omega: r(x)>0\}$.

The idea of the proof consists in breaking $\mathbf{R}^{k}$ into disjoint pieces and linking the estimates so obtained with the help of Lemma 5.1. Inside the bounded open set $B$, we apply the results of $\S 4$.B, where $r$ is allowed to be "singular"; outside $B$, we reduce the problem to $G_{\lambda}$ on which $r$ is "smooth" and positive and a method similar to that of $[\mathbf{F l}]$ can be used.

To prove Theorem 5.1, we shall need the following results:

PROPOSITION 5.1. There exist two positive constants $c^{\prime}$ and $c^{\prime \prime}$ such that, for all $\lambda$ sufficiently large,

$$
c^{\prime} \lambda^{k / 2 m}\left[G_{\lambda}\right] \leq \varphi(\lambda) \leq c^{\prime \prime} \lambda^{k / 2 m}\left[G_{\lambda}\right],
$$

where $\varphi(\lambda)=\int_{\Omega_{+}}\left[(\lambda r-q)_{+}\right]^{k / 2 m} \mu_{\mathcal{L}}^{\prime}$. 
PROOF OF PROPOSITION 5.1. It follows from (5.1)-(5.3) that

$$
\varphi(\lambda)=\int_{G_{\lambda}}(\lambda r-q)^{k / 2 m} \mu_{\mathcal{L}}^{\prime} \leq c^{\prime \prime} \int_{G_{\lambda}}(\lambda r)^{k / 2 m}
$$

and from (5.6) that

$$
\int_{G_{\lambda}}(\lambda r-q)^{k / 2 m} \mu_{\mathcal{L}}^{\prime} \geq \int_{G_{\lambda / 2}}(\lambda r-q)^{k / 2 m} \mu_{\mathcal{L}}^{\prime} \geq c^{\prime} \int_{G_{\lambda}}(\lambda r)^{k / 2 m}
$$

note that $r=r_{+}$and $\lambda r-q=(\lambda r-q)_{+}$on $G_{\lambda} \supset G_{\lambda / 2}$.

Proposition 5.2. If Theorem 5.1 holds for $N_{0}^{+}\left(\lambda ; r, \Omega, A^{\prime}\right)$, then it holds for $N_{0}^{+}(\lambda ; r, \Omega, \mathcal{A})$, where $\mathcal{A}^{\prime}=\mathcal{L}^{\prime}+q$ and $\mathcal{L}^{\prime}$ is the principal part of $\mathcal{L}$.

ProOF OF Proposition 5.2. This is a simple consequence of interpolation theorems and of Lemma 4.2 if we notice - as in [Ro 2, Lemma 1.1, p. 353]-that

$$
\varphi((1+\varepsilon) \lambda) \leq\left(1+c_{1} \sqrt{\varepsilon}\right)^{k / 2 m} \varphi(\lambda)
$$

for some positive constant $c_{1}$.

The result now follows by letting $\varepsilon \rightarrow 0$; here and thereafter, $\varepsilon$ denotes the variable used in Definition 5.1.

PROPOSITION 5.3.

$$
\begin{aligned}
& \lim _{\lambda \rightarrow+\infty} \lim _{\varepsilon \rightarrow 0} \varphi^{-1}(\lambda) \sum_{\zeta \in J} \varphi^{+}(\lambda, \zeta)=1 \\
& =\lim _{\lambda \rightarrow+\infty} \lim _{\varepsilon \rightarrow 0} \varphi^{-1}(\lambda) \sum_{\zeta \in I} \varphi^{-}(\lambda, \varsigma),
\end{aligned}
$$

where

$$
\begin{aligned}
\varphi^{ \pm}(\lambda, \zeta) & =\left[(1 \pm \varepsilon) \lambda r_{\zeta}-q_{\zeta}\right]^{k / 2 m} \mu_{\zeta}\left|Q_{\zeta}\right|, \\
q_{\zeta} & =q\left(x_{\zeta}\right) \quad \text { and } \\
\mu_{\zeta} & =(2 \pi)^{-k} \int_{\left\{\xi \in \mathbf{R}^{k}: \sum_{|\alpha|=|\beta|=m} a_{\alpha \beta}\left(x_{\varsigma}\right) \xi^{\alpha+\beta}<1\right\}} d \xi .
\end{aligned}
$$

This follows easily from hypothesis (5.5); observe that $\varepsilon \rightarrow 0$ implies that $\eta \rightarrow 0$.

PROOF OF THEOREM 5.1. In light of Proposition 5.2, it suffices to establish Theorem 5.1 for $A^{\prime}$.

Now, we make use of the covering $\left(Q_{\zeta}\right)_{\zeta \in \mathbf{z}^{k}}$ of $\mathbf{R}^{k}$ and of Lemma 5.1:

$$
\begin{aligned}
\sum_{\zeta \in I} N_{0}^{+} & \left(\lambda ; r, Q_{\zeta}, A^{\prime}\right) \leq N_{0}^{+}\left(\lambda ; r, \Omega, A^{\prime}\right) \\
\leq & N_{1}^{+}\left(\lambda ; r, B, A^{\prime}\right)+\sum_{\zeta \in J} N_{1}^{+}\left(\lambda ; r, Q_{\zeta}, A^{\prime}\right) \\
& +N_{1}^{+}\left(\lambda ; r, D, A^{\prime}\right)+N_{1}^{+}\left(\lambda ; r, G \backslash \bar{G}_{+}, \mathcal{A}^{\prime}\right),
\end{aligned}
$$

where $D=G_{+} \backslash\left(\bigcup_{\zeta \in J} \bar{Q}_{\zeta}\right)$.

Next, we remark that $N_{1}^{+}\left(\lambda ; r, G \backslash \bar{G}_{+}, A^{\prime}\right)=0$ because on $G \backslash \bar{G}_{+}, r$ is nonpositive and, by (4.6), there are no positive eigenvalues. Moreover, $N_{1}^{+}\left(\lambda ; r, D, A^{\prime}\right)=0$; the 
latter equality holds since $D \subset U_{\lambda}$, where $U_{\lambda}=\left\{x \in G_{+}: \lambda r(x)-q(x)<0\right\}$; hence $\left(\int_{U_{\lambda}} q|u|^{2} / \int_{U_{\lambda}} r|u|^{2}\right) \geq \lambda$ and, by $(4.6), N_{1}^{+}\left(\lambda ; r, U_{\lambda}, \mathcal{A}^{\prime}\right)=0$.

By Theorems 4.1 and 4.2, we have

$$
N_{i}^{+}\left(\lambda ; r, B, A^{\prime}\right) \sim \int_{B_{+}}\left[(\lambda r-q)_{+}\right]^{k / 2 m} \mu_{\mathcal{L}}^{\prime}, \quad \text { as } \lambda \rightarrow+\infty,
$$

where $B_{+}=\{x \in B: r(x)>0\}$ and $i=0$ or 1 according to the boundary conditions; in fact, by $(2.2)$, we may choose $R$ big enough so that $\left|B_{+}\right|>0$; moreover, we observe that, because $B$ is bounded,

$$
\int_{B_{+}}(\lambda r)^{k / 2 m} \mu_{\mathcal{L}}^{\prime} \sim \int_{B_{+}}\left[(\lambda r-q)_{+}\right]^{k / 2 m} \mu_{\mathcal{L}}^{\prime}, \quad \text { as } \lambda \rightarrow+\infty .
$$

It follows, in particular, that $\varphi^{-1}(\lambda) N_{i}\left(\lambda ; r, B, A^{\prime}\right) \rightarrow 0$ as $\lambda \rightarrow+\infty$.

Thanks to (5.7), we only have to work on $\bigcup_{\zeta \in J} Q_{\zeta}$, where $r$ is positively bounded from below by $\lambda^{-1} \gamma$ and satisfies $(\varepsilon)$. On each cube $Q_{\zeta}$, where $\varsigma \in J$, we can compare $A^{\prime}$ with a homogeneous operator with constant coefficients; precisely, for $i=0$ or 1 , it follows from condition $(\varepsilon)$ and from Lemmas 4.1 and 4.2 that, for $\eta$ small enough,

$$
\begin{aligned}
& N_{i}^{+}\left(\left[(1-\varepsilon) \lambda-q_{\zeta} / r_{\zeta}\right] ; r_{\zeta}, Q_{\zeta}, \mathcal{L}_{\zeta}\right) \leq N_{i}^{+}\left(\lambda ; r, Q_{\zeta}, A^{\prime}\right) \\
& \quad \leq N_{i}^{+}\left(\left[(1+\varepsilon) \lambda-q_{\zeta} / r_{\zeta}\right] ; r_{\zeta}, Q_{\zeta}, \mathcal{L}_{\zeta}\right),
\end{aligned}
$$

where $\mathcal{L}_{\zeta}=\sum_{|\alpha|=|\beta|=m} a_{\alpha \beta}\left(x_{\zeta}\right) D^{\alpha+\beta}$ and, as above, $r_{\zeta}=r\left(x_{\zeta}\right)$; furthermore, we know by a special case of [Me, Theorem 5.1, p. 175 and Theorem 5.12, p. 188] that there exists $C$ independent of $\varsigma$ such that

$$
\begin{gathered}
\left|N_{i}^{+}\left(\left[(1 \pm \varepsilon) \lambda-q_{\zeta} / r_{\zeta}\right] ; r_{\zeta}, Q_{\zeta}, \mathcal{L}_{\zeta}\right)-\mu_{\zeta}\right| Q_{\zeta}\left|\left[(1 \pm \varepsilon) \lambda r_{\zeta}-q_{\zeta}\right]^{k / 2 m}\right| \\
\leq C\left(\lambda r_{\zeta}\right)^{(k-1) / 2 m} .
\end{gathered}
$$

In light of Lemma 5.1, Propositions 5.1-5.3 and equations (5.7)-(5.10), we conclude the proof of Theorem 5.1 by choosing $\varepsilon=\lambda^{-1 / 2(2 m-1)}$ and by letting $\lambda \rightarrow+\infty$.

The next statement is really a corollary of the proof of Theorem 5.1 although it is an extension of Weyl's formula (Theorem 4.1).

THEOREM 5.2. If in assumption (5.2)(c) we suppose instead that $\int_{\Omega}|r|^{k / 2 m}$ is finite, we must replace the conclusion of Theorem 5.1 by that of Theorem 4.1:

$$
N^{+}(\lambda) \sim \int_{\Omega_{+}}(\lambda r)^{k / 2 m} \mu_{\mathcal{L}}^{\prime}, \quad \text { as } \lambda \rightarrow+\infty .
$$

REMARKS. 1. Of course, analogous estimates hold for negative eigenvalues provided that hypotheses (5.1), (5.2) and (5.5), (5.6) are changed accordingly.

It is noteworthy that the value of $\int_{\Omega}\left(r_{-}\right)^{k / 2 m}$ does not interfere with the asymptotics of $N^{+}(\lambda ; \mathcal{A}, r, \Omega)$ and vice versa. We give an example below where $N^{+}(\lambda)$ and $N^{-}(\lambda)$ do not have the same estimate (one is given by Theorem 5.1 and the other by Theorem 5.2). We note that, in the case when $\int_{\Omega}\left(r_{ \pm}\right)^{k / 2 m}$ are both finite, Theorem 5.2 (and its analogue for $N^{-}$) has been proved under slightly different hypotheses by Rozenbljum [Ro 1, Theorem 2, p. 246]. 
2. A result similar to Theorems 5.1 and 5.2 could also be obtained by the same methods for mixed Dirichlet-Neumann problems (under suitable compactness hypotheses).

3. Recently, Gurarie [Gu] has studied the asymptotic distribution of the eigenvalues of operators of Schrödinger type in $\mathbf{R}^{k}$ having a smooth positive weight function; in his work, based in part on the theory of pseudodifferential operators, the analogue of condition $(\varepsilon)$ is the so-called "finite propagation speed" condition.

The following result is of interest for certain applications:

PROPOSITION 5.4. The conclusions of Theorems 5.1 and 5.2 remain valid if we drop the hypothesis that $q$ tends to $+\infty$ at infinity and replace it by the assumption that $r$ is positive outside $B$.

In this case, as in the abstract part of $\S 4$, the imbedding of $V$ into $H$ is continuous (but not compact); however $T$ is compact by (5.4) since we still assume that $q^{-1} r$ tends to 0 at infinity.

EXAMPLE 5.1. We now illustrate our results by considering the following eigenvalue problem:

$$
A u=(-\Delta+q) u=\lambda r u, \quad \text { in } \mathbf{R}^{k}
$$

where

$$
r(x)= \begin{cases}-1 & \text { if }|x|<1 \\ 0 & \text { if }|x|=1 \\ +1 & \text { if }|x|>1\end{cases}
$$

and where $q$ satisfies (5.1) [e.g., $q(x)=\left(1+|x|^{2}\right)^{\sigma}$ with $\sigma \geq 1$ ].

According to Theorem 5.1, Theorem 5.2 and the preceding Remark 1, we then have

COROLlaRY 5.1.

$$
N^{-}(\lambda) \sim(2 \pi)^{-k}\left(B_{k}\right)^{2}|\lambda|^{k / 2}, \quad \text { as } \lambda \rightarrow-\infty,
$$

and

$$
N^{+}(\lambda) \sim(2 \pi)^{-k} B_{k} \int_{|x|>1}\left[(\lambda-q(x))_{+}\right]^{k / 2} d x, \quad \text { as } \lambda \rightarrow+\infty,
$$

where $B_{k}$ denotes the volume of the unit ball in $\mathbf{R}^{k}$.

NOTE ADDED IN PROOF. Because of an erroneous numerical choice of the sharp Sobolev constant [LY, equation (19), p. 316], the claim in [LiY, Theorem 2 and Corollary 2] that the constant found there is better than in [Lb] is not justified. The best constant for the Cwikel-Lieb-Rozenbljum bound seems still due to Lieb in [Lb], as is clearly discussed on page 475 of E. H. Lieb, On characteristic exponents in turbulence, Comm. Math. Phys. 92 (1984), 473-480. In §3.C of the present paper, one should therefore use [Lb, Theorem 2, p. 243] rather than [LiY, Theorem 2, p. 314]; more specifically, if $L_{k}$ and $\tilde{C}_{k}$ are defined as in [Lb, p. 243] and [LiY, p. 310], respectively, one must replace $\Theta_{k}$ by $\tilde{C}_{k}=\left(L_{k}\right)^{-2 / k}$ in equation (3.10) of Theorem 3.3 and substitute $L_{k}^{-1} N^{ \pm}(\lambda)$ for the left-hand side of the inequality in Corollary 3.2.

We wish to thank Professor Elliott H. Lieb for bringing these facts to our attention. 
ACKNOWLEDGement. We wish to thank Argonne National Laboratory which made this collaboration possible. Jacqueline Fleckinger is grateful to the University of Southern California for its hospitality and to the organizers of the International Conference at Pan Am University (Texas). Michel L. Lapidus is indebted to the Mathematical Sciences Research Institute at Berkeley of which he was a member while part of this paper was completed and to the Institute for Mathematics and its Applications at Minneapolis for its hospitality in November 1984.

Both authors are thankful to E. Brian Davies for his remarks and to Didier Robert and Barry Simon for some references.

\section{REFERENCES}

[Ad] R. A. Adams, Sobolev spaces, Academic Press, New York, 1975.

[Ag] S. Agmon, Lectures on elliptic boundary value problems, Van Nostrand, Princeton, N. J., 1965.

[Be] R. Beals, Indefinite Sturm-Liouville problems and half-range completeness, J. Differential Equations 56 (1985), 391-407.

[BS 1] M. S. Birman and M. Z. Solomjak, Spectral asymptotics of nonsmooth elliptic operators. II, Trans. Moscow Math. Soc. 28 (1973), 1-32.

[BS 2] _ Asymptotics of the spectrum of variational problems on solutions of elliptic equations, Siberian Math. J. 20 (1979), 1-15.

[BS 3] , Asymptotic behavior of the spectrum of the differential equations, J. Soviet Math. 12 (1979), 247-282.

[BS 4] _ Quantitative analysis in Sobolev imbedding theorems and applications to spectral theory, Amer. Math. Soc. Transl. (2) 114 (1980).

[Bo] M. Bôcher, Boundary problems in one dimension, Proc. Fifth Internat. Congress Math. (Cambridge, 1912), Vol. I, Cambridge Univ. Press, New York, 1913, pp. 163-195.

[Br] F. E. Browder, Le problème des vibrations pour un opérateur aux dérivées partielles selfadjoint et du type elliptique à coefficients variables, C. R. Acad. Sci. Paris Sér. A 236 (1953), 2140-2142.

[CH] R. Courant and D. Hilbert, Methods of mathematical physics, Vol. I; English transl., Interscience, New York, 1953.

[DGHa] I. Dee Chang, G. W. Grube and E. Y. Harper, On the breakup of accelerating liquid drops, J. Fluid Mech. 52 (1972), 565-591.

[dF] D. G. de Figueiredo, Positive solutions of semilinear elliptic problems, Lecture Notes in Math., vol. 957, Springer-Verlag, Berlin, 1982, pp. 34-87.

[Fl] J. Fleckinger, Estimate of the number of eigenvalues for an operator of Schrödinger type, Proc. Roy. Soc. Edinburgh Sect. A 89 (1981), 355-361.

[FlF 1] J. Fleckinger and M. El Fetnassi, Asymptotics of eigenvalues of variational elliptic problems with indefinite weight function, Spectral Theory of Sturm-Liouville Differential Operators (Hans G. Kaper and A. Zettl, eds.), ANL-84-73, Argonne National Laboratory, Argonne, Illinois, 1984, pp. 107-118.

[F1F 2] _ Comportement asymptotique des valeurs propres de problèmes elliptiques "non définis à droite”, C. R. Acad. Sci. Paris Sér. I Math. 299 (1984), 599-602.

[FIMe] J. Fleckinger and G. Métivier, Théorie spectrale des opérateurs uniformement elliptiques sur quelques ouverts irréguliers, C. R. Acad. Sci. Paris Sér. A 276 (1973), 913-916.

[Ga] L. Gårding, The asymptotic distribution of the eigenvalues and eigenfunctions of elliptic operators, Math. Scand. 1 (1953), 55-72.

[GN] W. N. Gill and R. J. Nunge, Analysis of heat or mass transfer in some countercurrent flows, Internat. J. Heat Mass Transfer 8 (1965), 873-886.

[GoK] I. C. Gohberg and M. G. Krein, Theory and applications of Volterra operators in Hilbert spaces, Transl. Math. Monographs, vol. 24, Amer. Math. Soc., Providence, R.I., 1970.

[Gu] D. Gurarie, $L^{p}$ and spectral theory for a class of global elliptic operators on $\mathbf{R}^{n}$, Preprint \#84-10, Case Western Reserve Univ., Dept. of Mathematics and Statistics, 1984. 
[H] P. Hess, On bifurcation from infinity for positive solutions of second order elliptic eigenvalue problems, Nonlinear Phenomena in Mathematical Sciences, Academic Press, New York, 1982, pp. 537-544.

[HK] P. Hess and T. Kato, On some linear and nonlinear eigenvalue problems with an indefinite weight function, Comm. Partial Differential Equations 5 (1980), 999-1030.

[Hi] D. Hilbert, Grundzüge einer allgemeinen Theorie der linearen Integralgleichungen, Teubner, Leipzig, 1912; Chelsea, New York, 1953.

[Ho] E. Holmgren, Über Randwertaufgaben bei einer linearen Differentialgleichung zweiter Ordnung, Ark. Mat., Astro och Fysik 1 (1904), 401-417.

[Kc] M. Kac, Can one hear the shape of a drum?, Amer. Math. Monthly (Slaught Memorial Papers, No. 11) (4) 73 (1966), 1-23.

[KKLeZ] H. G. Kaper, M. K. Kwong, C. G. Lekkerkerker and A. Zettl, Full- and half-range eigenfunctions expansions for Sturm-Liouville problems with indefinite weights, Proc. Roy. Soc. Edinburgh Sect. A 98 (1984), 69-88.

[KZ] H. G. Kaper and A. Zettl (eds.), Proc. May-June 1984 Workshop "Spectral Theory of SturmLiouville Differential Operators", ANL-84-73, Argonne National Laboratory, Argonne, Illinois, 1984.

[La 1] M. L. Lapidus, Valeurs propres du laplacien avec un poids qui change de signe, C. R. Acad. Sci. Paris Sér. I Math. 298 (1984), 265-268.

[La 2] _ Spectral theory of elliptic problems with indefinite weights, Spectral Theory of Sturm-Liouville Differential Operators (Hans G. Kaper and A. Zettl, eds.), ANL-84-73, Argonne National Laboratory, Argonne, Illinois, 1984, pp. 159-168.

[LiY] P. Li and S. T. Yau, On the Schrödinger equation and the eigenvalue problem, Comm. Math. Phys. 88 (1983), 309-318.

[Lb] E. Lieb, The number of bound states of one-body Schrödinger operators and the Weyl problem, Proc. Sympos. Pure Math., vol. 36, Amer. Math. Soc., Providence, R.I., 1980, pp. 241-252.

[LM] J. L. Lions and E. Magenes, Non-homogeneous boundary value problems and applications, vol. I, English transl., Springer-Verlag, Berlin, 1972.

[LoS] L. H. Loomis and S. Sternberg, Advanced calculus, Addison-Wesley, Reading, Mass., 1968.

[LuR] G. S. S. Ludford and R. A. Robertson, Fully diffused regions, SIAM J. Appl. Math. 25 (1973), 693-703.

[LuW] G. S. S. Ludford and S. S. Wilson, Subcharacteristic reversal, SIAM J. Appl. Math. 27 (1974), 430-440.

[MM] A. Manes and A. M. Micheletti, Un'estensione della teoria variazionale classica degli autovalori per operatori ellittici del secondo ordine, Boll. Un. Mat. Ital. 7 (1973), 285-301.

[Me] G. Métivier, Valeurs propres de problèmes aux limites elliptiques irréguliers, Bull. Soc. Math. France Mém. 51-52 (1977), 125-219.

[Pe] I. N. Pesin, Classical and modern integration theories, English transl., Academic Press, New York, 1970.

[Pl] Å. Pleijel, Sur la distribution des valeurs propres de problèmes régis par l'équation $\Delta u+$ $\lambda k(x, y) u=0$, Ark. Mat., Astr. och Fysik 29 B (1942), 1-8.

[RS] M. Reed and B. Simon, Methods of modern mathematical physics, Vol. IV, Analysis of operators, Academic Press, New York, 1978.

[Ri] R. G. D. Richardson, Contributions to the study of oscillation properties of the solutions of linear differential equations of the second order, Amer. J. Math. 40 (1918), 283-316.

[Ro 1] G. V. Rozenbljum, The distribution of the discrete spectrum for singular differential operators, Soviet Math. Dokl. 13 (1972), 245-249.

[Ro 2] _ Asymptotics of the eigenvalues of the Schrödinger operator, Math. USSR-Sb. 22 (1974), 349-371.

[Ro 3] _ Asymptotics of the eigenvalues of the Schrödinger operator, Problemy Mat. Anal. Slož. Sistem. 5 (1975), 152-166. (Russian)

[T] E. C. Tichmarsh, Eigenfunction expansions associated with second-order differential equations, Part II, Oxford Univ. Press, London, 1958.

[Wn] H. F. Weinberger, Variational methods for eigenvalue approximation, CBMS Regional Conf. Ser. Appl. Math., vol. 15, SIAM, Philadelphia, Penn., 1974. 
[We] H. Weyl, Das asymptotische Verteilungsgesetz der Eigenwerte linearer partieller Differentialgleichungen, Math. Ann. 71 (1911), 441-469.

[Y] S. T. Yau, Survey on partial differential equations in differential geometry, Seminar on Differential Geometry (S. T. Yau, ed.), Ann. of Math. Studies, no. 102, Princeton Univ. Press, Princeton, N.J., 1982, pp. 3-71.

DePaRtement de MAThematiques 1R2, Universite PAUl SABATIER, 118 Route de NARBONNE, 31062 TOUlOUSE, CEDEX, FRANCE (Current address of Jacqueline Fleckinger)

Mathematical Sciences Research Institute, Berkeley, CaliforNia 94720

Current address (M. L. Lapidus): Department of Mathematics, DRB 306, University of Southern California, Los Angeles, California 90089-1113 